\title{
The Analysis on the Fundamental Influence of the Internet Thinking on the Art Production
}

\author{
Zhe $X u^{1, a}$ \\ ${ }^{1}$ Literature College of Jinan University, Guangzhou, Guangdong, China, 510632 \\ azilanbingmo@126.com
}

Keywords: Internet Thinking, Consumers, Digitization, Art Production

\begin{abstract}
Recently, though the elite of all walks of life have a different view about the Internet thinking, in general, the Internet thinking refers to an open industry model based on the network. This thinking has impact on the traditional cultural industry model, and has brought new changes to the development of the cultural industry. These new changes will inevitably affect the development of literature and art, which would bring new vitality to the development of literature and art.
\end{abstract}

\section{Introduction}

The information technology revolution of which the core is the Internet is sweeping people's lives. And the burgeoning electricity supplier, literature, music and films and other cultural products take the network as the carrier to spread, which make the Internet become an important tool in daily life . Given that the connection between Internet and people's daily life has been more and more close. Baidu CEO Robin Li first proposed the "Internet thinking", a new vocabulary of Science and Technology. New things always cause a variety of arguments, some support and others opposed. while this "Internet thinking" attracts scholars, social elites to define and express their views. Zhou Hongyi, the chairman of 360, summed up the Internet thinking as "putting customer at the center, the power of experience, free business model, subversive innovation." (2014-10) Haier's Zhang Ruimin believes that the Internet thinking "should be zero distance, network of thinking "(2014-1). In "A Book to Understand Internet Thinking", Pang Xiaolong wrote down this definition of "Internet thinking": "The Internet can be said to be a human thinking, pay more attention to human values in the world of the Internet, the customer becomes the God, the previous business model is no longer used, and now enterprises should pay more attention to customers' need, and view the customer as God."(2014, p16)

The entrepreneurs cognizes the Internet thinking focused on the relationship between producers, consumers and products, and summarizing the definition of the Internet the scholars and businessmen have presented, we can drawn the basic characteristics of the Internet thinking: putting customer at the center, digitization, more open mode. These characteristics can impact the inherent business model and we can say the Internet thinking involved in economic production and consumption, which must be able to drive a new industrial development model and produce economic benefits. And also some scholars like Professor Jin Yuanpu been concerned about the impact of the Internet thinking in economic would be bound to affect the social thinking, values and philosophy paradigm, "Internet thinking is the pilot concept about the third industrial revolution, which is the new ways of thinking that produced by cross-border integration of high-tech and contemporary culture, and which is the inevitable outcome of the paradigm shift in the revolution of science and technology. It has its own unique properties and mode of operation and would reinterprete the philosophy of all social life generally linked the highest standards under the realistic conditions and technological development. It indicates, a newer way of thinking is shaping, which will bring more changes in the future of society. "(Jin Yuanpu, p42) The Renaissance and the spinning machine is a good example. The advances of technology promote the transformation of the economic model and the growth of economic benefit. And the economic basement determines the superstructure, and thus technological innovation not only bring productivity change, it can also cause changes in all aspects of society, naturally, the ideological field. 
Literature and art is an important part of the ideology, which must be influenced by Internet thinking. Internet thinking is involved in the cultural industry, which would change the original industrial model of literature and art, such as from bookstores to e-books, which would affect the development of literature and art. In the above, we mentioned the important feature of Internet thinking: putting customer at the center, digitization, more open mode, which will affect the production, distribution, and consumption of the literary arts industry, thereby affecting the development of literature and art itself.

\section{Putting customer at the center}

In the three characteristics of the Internet thinking, the core of which is consumer-oriented, because in the Internet era, product design, video news are all born by all users on the Internet. Consumers click mouse and generated the consumer behavior, which is the source of economic benefit. Consumers in the Internet world is completely out of choice or click on their own thinking and consciousness, which is completely autonomous behavior, and it is not like in store, no shopping guide and other commodities to introduce and affect consumer choice. Internet users are completely autonomous. And to attract consumers, the sellors must take the consumers needs as highest guidelines. Respecting consumer demand not only able to attract consumer spending, more importantly, the user experience is no longer a simple small-scale evaluation or private chat in the Internet era, users reviews played a role beyond advertising, a praise for businesses is possible to attract more consumers, because these "praise" is based on the users' own experience, which has a star effect than advertising. Consumer groups are ordinary people, naturally, consumers are more willing to believe the view from the men like themselves. Therefore, to develop the cultural industry in the Internet age, the users' experience must be respect.

At the same time, the Internet does offer a platform to provide advice from consumers in the development of cultural industries. A variety of network platforms: micro-blog, blog, Post Bar, which are the sources of big data. On the internet, consumers use simple words, pictures or videos of themselves to express teir own view about something or some kind of cultural commodity. Among them, especially the movies, television dramas and variety shows, are the hot topic on the various platforms. So do the literature, in the network literary creation platform, authors and readers would have a lively discussion, sometime the authors even draw readers' recommendations in the creation and modified the text, while the rank or score is able to attract more readers, and based on the hits, the writers can have income. The same as the music, Chinese music charts, the song list and so on, which based the Internet users' choice or hits to rank. These consumers' choice reflected the cultural needs of user, the user is respected. Perhaps in the near future, cultural production mode will become such mode that the audiences vote determine an item's appearance and the content, and then product them and spread by the network, and then continuous improvement under the consumers' view. The economic benefits will be produced in series of segments. According to the current development of the Internet, this conjecture will come true one day. In these processes, the characteristics of traditional literature and art are bound to change.

Some might say that these commodities become literary art is public, but not enough elegance. Network literature is not the elegant works of literature, but "The Three Musketeers", "La Traviata" in their ages, were just spread in popular, their classical meaning was presented with the time going, the classic baptism is inseparable from the time and the selection, we cannot simply conclude that now some of the popular Internet novel or other forms of literature that cannot be defined as the vulgar literature. In these networks literature, just as mentioned above, it is no longer a traditional literary author who decides everything, creative works become open, the reader can also involved the creation of literature.

Jauss has proposed to establish a history of accepting literary based on the readers of literature, and this history of literature can truly reflect the relationship among times, readers and literary works, but this propose have not put into practice. But after Internet thinking involved in the development of literature and art, the assumption becomes feasible, borrow from big data, we can analyze the needs of most users or evaluation, whether the literary acception history or the 
acception history of moive, they all can be established.

In short, because the Internet thinking of the supremacy of users, readers, viewers, listeners and other literature and art recipients became the center of literary and artistic creation. Although the artists have tried to pursue independent pure artistic creation, they can inevitable be affected in this environment. The popular mindset and values have an increasingly important influence in the literary and artistic creation. Therefore, we can say, under the influence of the Internet thinking, the acceptors of literature and art are playing an increasingly important role.

\section{Digitization}

Internet thinking is based on Internet technology and digital technology, so the digitization is the basic elements in Internet thinking. With the development of science and technology, digital spread of commodity production, a lot goods take entity as the carrier gradually changed to digital technology as the carrier, e-books have take most part of the book market, while the carrier of music and movies also from DVD and CD discs into a digital network.

In literature and art industry, many works of art have been digitized, not only in the production process but also the carrier, and the more important is that the speed and breadth of the spread of digital artwork is much higher than the entity carrier of art. One might think that only these public artworks could adapt to the digital age, however, it is not the case, in the field of arts, music, painting, music and dance can be a better means of digital models, and literary works too. Painting is the art that need entity tools to creat, like pen and paper, it may not adapt to digital media. While, it is not true, the the micro-channel public account National Museum provides a digital platform for the dissemination of the exhibition of calligraphy and painting. It can be seen that the Internet thinking have been involved into the traditional cultural industry model, and have changed the traditional cultural carrier already.

Not only does the Internet thinking convert the culture industry carriers and forms of communication but also change the cultural industry model. The music, literature, film and television have broken the traditional barriers and gradually fused together through the industrial chain. For example, after the popular network literature, "Zhen Huan Biography", being adapted to the TV series, it also spread online, as well as the theme song for the TV series episode is also popular on the network. Under the influence of the Internet thinking, such works will be more closely integrated with various art form to be an industy chain established on the network.

Overall, the internet thinking have an influence on changing the traditional thinking in the cultural industry model, at the same time, it bound to affect the production and development of literary and artistic works. Digital technology has changed the carrier and the dissemination of literary and artistic works, infiltrated into the literary and artistic works, and break the barriers among different artistic fields, so that the various arts can be interconnected learn from each other. Since the birth of these art categories, they have been in mutual relations, and under the influence of the Internet thinking, these categories can be in better relationship. Meanwhile, the penetration of digital to the artwork will inject new elements for the creation of art, the film has already digitized baptism, other works of art will certainly show some new creative features because of the emergence of digital. The relationship between art and technology have had in the history s the best example to prove that Internet thinking appears would have an impact on the literary and artistic creation, like the emergence of the avant-garde.

Therefore, the Internet thinking would have an impact of production literary and artistic, not only on the carrier, the media is also reflected the relationship between the various disciplines of the arts or creative approach.

\section{The More Open Model}

In the traditional business model, for consumers, from production to consumption, they can contact only ultimate style goods, but after the Internet thinking involved in business model, consumers have access to all aspects of the production, so does the culture industry. March 26, 2014, 
Alibaba and Guohua Life Insurance created a crowdfunding platform named "Yulebao"(the treasure of entertainment) toghter, and consumers can be invested $100 \mathrm{RMB}$ to take part in the film making. The movies participate in the value-added platform included: " Tiny Times 3", "Wolf Totem" and so on. And according to box office, it will pay for corresponding benefits. While the the box office defeat of " Cribug III" let Ali "Yulebao" underperformed. And then, in September, Baidu also launched the "Baifayouxi", the same crowdfunding platform as "Yulebao". They raise funds for the moive " The Golden Era ". "Baifayouxi” set 10 yuan as the minimal investment, and the payment to investors is related to box office too. However, as the " The Golden Era " cannot meet the expectations of the audience, the investors payment can't be guaranteed, at last, Baidu's "Baifayouxi" also lost in the first battle.

For many ordinary consumers, such crowd funding, they invested 100 yuan or 10 yuan perhaps not for making money, but as a film fan's curiosity and participation mentality. After all, for ordinary consumers, the investment for movies or games are always from the rich, but now they can also have such experience as an investor, this experience is the most important. Furthermore, the reason why Alibaba and Baidu first established investment platform failed is that the idea is not good but the moives involved in the projects are not attract enough audience. So what decide the both creative industry projects to be well or not is the quality of the film.

Although the progress of these two creative projects is not particularly smooth, but as emerging things, they will inevitably encounter some ups and downs, It is important that their presence conforms to the development of the Internet thinking and they are an attempt to change the business model under the new technology in the Internet era. Although in the initial stages, the economic benefits is not satisfactory, but what these projects reflected is significant. First, the audience and the consumer have been in a passive position for long time in production activities but now the position began to change influenced by the science and technology. In the traditional industry model, consumers intervene commercial chain just on the stage of merchandise sales, but the new industry model will allow consumers to take part in the chain of production and consumption, so the views of the audience will play a greater role, which will change the traditional model of literary and artistic creation, and the individual consciousness of authors, directors, writers and other literary and artistic producer can no longer be completely dominant the production of literary and art, and their opinions, views perhaps can not fully reflected in the literary or art workst, but to refer to public opinion, especially the films, the popular art. Secondly, the "Yulebao" "Baifayouxi", its propaganda value of cultural products is greater than the value of funds raised, although it is claim to raise funds for the movies the real effect is to attract movie audience, in this case, the film is not rely on the storyline to attract the audience, actors and other things are more attractive. For narrative art-film, this is a great influence and change. Of course, now, the impact the Internet thinking have on the traditional art forms like literature is much less than the movies, because movies and TV shows, after all, is the public art and high investment cost-effective cultural products. But from the network literature, the influences can be seen.

\section{Conclusions}

In short, Internet thinking involved in cultural industries has brought challenge to the traditional model and resulting in new elements. These new factors will affect the cultural products, thereby affect the development of literature and art. Based on the analysis, we can sum up some of specific performance that the the Internet thinking impact the development of literary and artistic. Because readers, viewers and other identities have been improved in business activities and they take part in the art producation more and more activity, which would allow acceptors to give artistic values and specific view to literature and art creators, and then change the pattern of the original art. Secondly, the form and carriers of literary have changed already, digitization not only enhances the speed of their spread but also changes the form of literature and art. Under the national support, the Internet thinking is bound to change the traditional cultural model in a broader and deeper range which would ultimately affect the further development of literature and art. 


\section{References}

[1] Wang Zhiyan, Zhou Hongwei. Internet Thinking Is the Return of Common Sense. China Youth Daily, 2014-10-8(10).

[2] He Cheng, Zhang Ruimin: Haier Operating under Internet Thinking Do Not Need What Kind of Person. http://money.163.com/14/0120/06/9J0U5E5F00253B0H.html.

[3] Pang Xiaolong. A Book to Understand Internet Thinking. Changchun: JiLin Publishing Group Co. Ltd, 2014.

[4] Jin Yuanpu. Internet Thinking: Paradigm Change in the Era of Science and Technology Revolution. Fujian Tribune (The Humanities \& Social Sciences Bimonthly), (2014) No 10, p.42-48. 